\title{
CONSUMO DE ANTIMICROBIANOS EM UNIDADES DE TERAPIA INTENSIVA NEONATAIS: RESISTÊNCIA BACTERIANA X AÇÕES DE RACIONALIZAÇÃO
}

\author{
ANTIMICROBIAL CONSUMPTION IN NEONATAL INTENSIVE CARE UNITS: \\ BACTERIAL RESISTANCE X RATIONALIZATION ACTIONS
}

\author{
Cinara Rejane Viana Oliveira
}

Universidade do Estado da Bahia

Abstract

Antimicrobials are among the most prescribed drugs in Neonatal Intensive Care Units (NICU). Thus, greater control in its use is necessary, mainly due to the increase in bacterial resistance. The "Antimicrobial Stewardship Program" is an antimicrobial management program that consists in a set of coordinated interventions with the aim of improving and measuring the appropriate use of antimicrobials. In Brazil, the "Gerência de Vigilância $e$ Monitoramento em Serviços de Saúde", with the "Gerência Geral de Tecnologia em Serviços de Saúde", and the "Agência Nacional de Vigilância Sanitária", in adaptation of the mentioned program to the reality of Brazil, created the "Diretriz Nacional para Elaboração de Programa de Gerenciamento do Uso de Antimicrobianos em Serviços de Saúde". In it, there are actions that must be implemented in health units that aim the rational use of antimicrobials. Considering the need for measures in this direction, this work will address actions described in the academic literature that can be implemented in the context of Neonatal Intensive Care Units aiming to reduce the incorrect use of antimicrobials.

Keywords: Antimicrobials; Neonatology; Bacterial Resistance.
Resumo

Os antimicrobianos estão entre os medicamentos mais prescritos nas Unidades de Terapia Intensiva Neonatais (UTIN). Assim, é necessário um maior controle em sua utilização devido principalmente ao favorecimento do aumento da resistência bacterina. $O$ "Antimicrobial Stewardship Program" é um programa de gerenciamento de antimicrobianos que consiste em um conjunto de intervenções coordenadas, com o objetivo de melhorar e medir o uso adequado dos antimicrobianos. No Brasil, a Gerência de Vigilância e Monitoramento em Serviços de Saúde, em conjunto com a Gerência Geral de Tecnologia em Serviços de Saúde, e a Agência Nacional de Vigilância Sanitária, adaptando o citado programa à realidade do Brasil, elaborou a Diretriz Nacional para Elaboração de Programa de Gerenciamento do Uso de Antimicrobianos em Serviços de Saúde. Nessa diretriz estão abordadas ações que devem ser implantadas nas unidades de saúde que visam ao uso racional de antimicrobianos. Considerando a necessidade de atenção nesse contexto, esse trabalho irá abordar ações que visem à redução do uso incorreto de antimicrobianos descritas na literatura e que podem ser implantadas no contexto das Unidades de Terapia Intensiva Neonatais.

Palavras-chave: Antimicrobianos; Neonatologia; Resistência Bacteriana. 
Entre os medicamentos mais utilizados nas Unidades de Terapia Intensiva Neonatais (UTINs) encontram-se os antimicrobianos (ATM). ${ }^{1}$ O uso excessivo de antimicrobianos especialmente em bebês prematuros em UTINs pode resultar em consequências em nível de unidade e de saúde pública, como o surgimento de organismos cada vez mais resistentes a vários medicamentos o que acaba repercutindo a maiores custos de saúde. ${ }^{2-3}$

$O$ aumento de agentes infecciosos multirresistentes repercute na utilização de uma antibioticoterapia prolongada, empírica e que seja amplamente eficaz em recém-nascidos assim, as indicações para tratamentos com antibióticos devem ser analisadas mais criticamente e, se necessário, serem submetidas a uma reavaliação de base infecciosa pois, o uso excessivo e inadequado de antimicrobianos pode levar ao surgimento de organismos resistentes resultando em efeitos adversos neonatais. ${ }^{4-5}$

O uso prolongado de antimicrobianos de amplo espectro, especialmente em neonatos de alto risco, tem sido apontado como fator de risco para a ocorrência de enterocolite necrosante, displasia broncopulmonar, candidíase invasiva e até mesmo morte. ${ }^{6-7}$

A resistência antimicrobiana tornou-se uma preocupação mundial, uma vez que as bactérias estão mais letais e estudos direcionados a descobertas de novos antimicrobianos de amplo espectro são escassos. Com o intuito de reduzir a resistência bacteriana, vários países estão criando políticas que visam o uso sustentável dos ATM. ${ }^{8}$

Em neonatologia é fundamental a implementação de intervenções estruturadas de manejo antimicrobiano além do desenvolvimento de outras ações de saúde que priorizem o seu uso de forma racional. ${ }^{9}$

Devido à necessidade da ampliação do conhecimento em neonatologia e sobre utilização de ATM nesse público, esse estudo tem como objetivo realizar uma revisão sobre possíveis ações que podem ser implantadas e que são descritas na literatura com o intuito de reduzir o uso incorreto de antimicrobianos no contexto das Unidades de Terapia Intensiva Neonatais.
O presente estudo trata-se de uma revisão narrativa de literatura, no qual, para descrever a metodologia foram definidas as seguintes etapas: identificação da questão norteadora; estabelecimento dos critérios de inclusão e exclusão do estudo; avaliação dos estudos incluídos; interpretação dos resultados; apresentação da revisão/ síntese do conhecimento.

Foi levantada a seguinte questão norteadora para concretização da pesquisa: “Os programas de gerenciamento de antimicrobianos são eficazes para diminuir o consumo de antimicrobianos em UTIS neonatais?".

A coleta de dados foi realizada utilizando-se as bases de dados: National Center for Biotechnology Information (PUBMED), Scientific Electronic Library Online (SCIELO) e a Literatura Latino-americana e do Caribe em Ciências da Saúde (LILACS).

Os descritores selecionados para a busca dos artigos foram: stewardship, antimicrobianos e Unidades de Terapia Intensiva Neonatais. O operador boreano "AND" foi utilizado para melhor selecionar os artigos.

Como critérios de inclusão, utilizaramse artigos originais publicados entre os anos de 2010 e 2021, disponíveis na íntegra, que abordaram a temática antimicrobianos em neonatologia/utilização de programas de gerenciamento. Os critérios de exclusão envolveram as pesquisas que contemplassem a utilização de antimicrobianos em indivíduos que não eram pediátricos ou neonatais. Os artigos foram triados entre os meses de dezembro de 2020 e maio de 2021, utilizando os descritores nos idiomas inglês e português.

$$
\text { Para seleção dos estudos }
$$
primeiramente foi realizada a leitura dos títulos e resumos e conseguinte a leitura na integra dos que se adequavam a temática. Por fim realizou-se a síntese dos estudos abordando os pontos que mais se adequavam. 
Figura 1 - Fluxograma das referências utilizadas no estudo

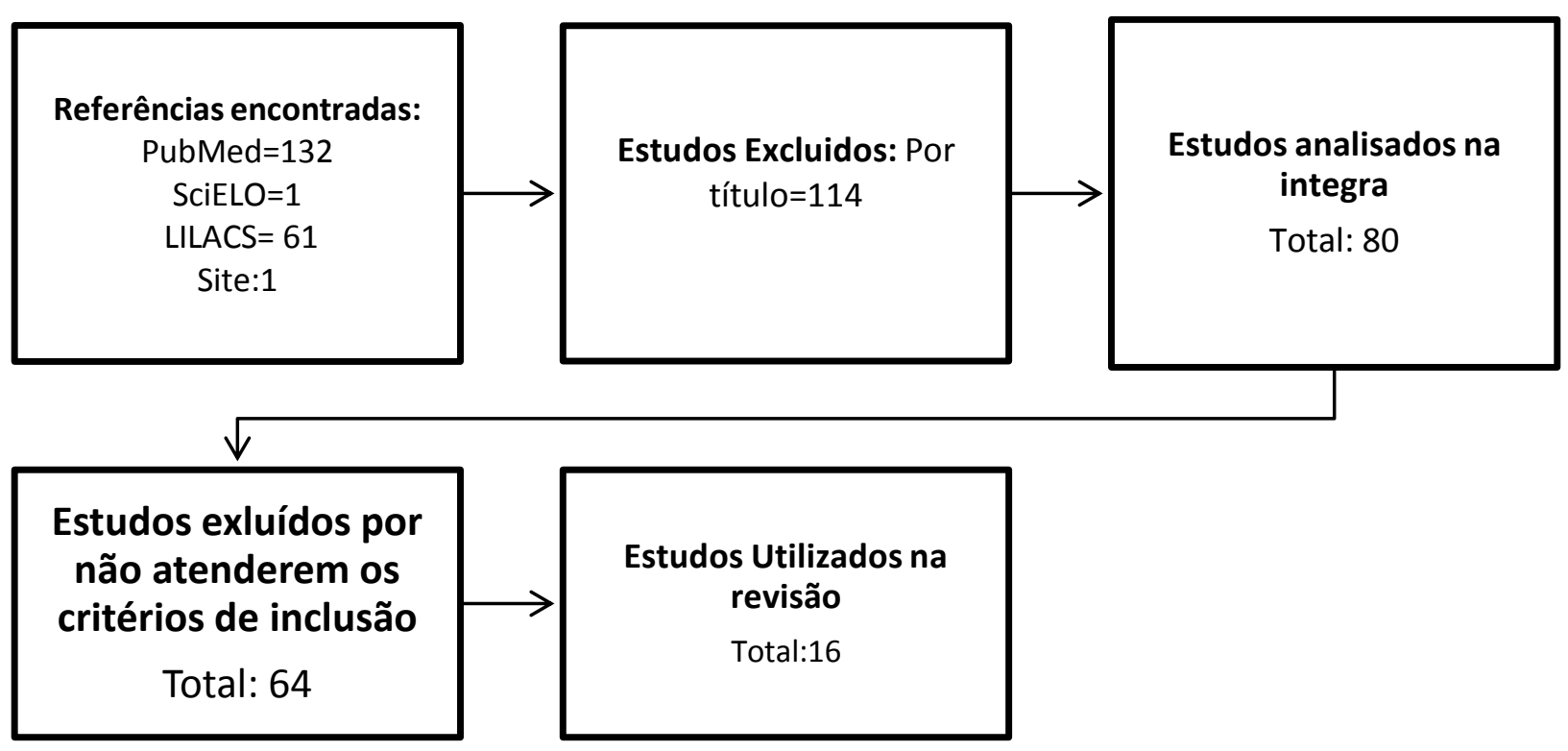




\section{Resultados e Discussão}

Programa de gerenciamento de antimicrobianos

O "Antimicrobial Stewardship Program (ASP)" é um programa de gerenciamento de antimicrobianos que consiste em um conjunto de intervenções coordenadas, com a função de melhorar e medir o uso adequado dos antimicrobianos por meio da promoção da seleção otimizada do regime antimicrobiano ideal. $^{10}$

Os principais objetivos do ASP consistem em elaborar medidas de prevenção e controle de infecção, para prevenir ou retardar o aparecimento da resistência antimicrobiana e a transmissão de agentes patogênicos antimicrobianos resistentes. Várias estratégias são aplicadas para melhorar o tratamento com antimicrobianos, diminuir as consequências não intencionais e aperfeiçoar os resultados clínicos. Com a aplicação dessas medidas consequentemente têm-se a redução dos custos dos cuidados de saúde sem prejudicar sua qualidade. ${ }^{11}$

A Gerência de Vigilância e Monitoramento em Serviços de Saúde (GVIMS), da Gerência Geral de Tecnologia em Serviços de Saúde (GGTES), da Agência Nacional de Vigilância Sanitária (ANVISA), adaptando à realidade do Brasil elaborou a Diretriz Nacional para Elaboração de Programa de Gerenciamento do Uso de Antimicrobianos em Serviços de Saúde, seguindo o arcabouço conceitual e técnico operacional do ASP.

São necessários alguns elementos essenciais para a implantação e execução desse programa nos hospitais como: apoio da alta direção do Hospital; definição de responsabilidades de todos os profissionais envolvidos; educação; desenvolvimento de ações para melhorar a prescrição de antimicrobianos; monitoramento do programa e divulgação de resultados. ${ }^{12}$

A Diretriz Nacional para Elaboração do Programa de Gerenciamento do Uso de Antimicrobianos em Serviços de Saúde reporta que as ações de gerenciamento de antimicrobianos, vão desde abordagens educativas até medidas restritivas, entre elas encontram-se: utilização de protocolos clínicos para as principais síndromes clínicas; adoção das boas práticas de prescrição, como documentação de dose, duração e indicação do antimicrobiano; auditoria prospectiva de prescrição com intervenção e divulgação dos dados; readequação da terapia, conforme resultados microbiológicos; análise técnica das prescrições pela farmácia; restrição com uso de formulário terapêutico e pré-autorização de antimicrobianos. $^{12}$

Nessa revisão serão abordadas três dessas ações, serão elas: utilização de protocolos clínicos para as principais síndromes clínicas, auditoria prospectiva de prescrição com intervenção e divulgação dos dados e análise técnica das prescrições pela farmácia. A discussão será direcionada às Unidades de Terapia Intensivas Neonatais, demonstrando através de estudos a importância da implantação dessas ações.

Utilização de protocolos clínicos para as principais síndromes clínicas

A implantação nas unidades de saúde de protocolos clínicos baseados em evidências científicas e em práticas de consensos é de suma importância para os profissionais de saúde, técnicos e gestores, pois, ocorre a padronização de suas atividades. ${ }^{12}$

As unidades de saúde devem adaptar a sua realidade durante a confecção e aplicação dos protocolos, identificando as características clínicas e os perfis epidemiológicos e microbiológicos locais. Antes da implantação é importante analisar se existem guias regionais ou nacionais para adaptá-los às condições epidemiológicas, recursos de diagnóstico e arsenais terapêuticos institucionais. ${ }^{12}$

Para melhor entendimento, os protocolos devem ser claros e serem atualizados periodicamente para assim reduzir a diferença entre as condutas dos profissionais e melhorar a avaliação de processos e resultados, pois, consequentemente aumenta diretamente a qualidade e a segurança da assistência. $^{12}$

A adoção de protocolos de uso de antimicrobianos é efetiva e proporciona a promoção do uso correto desses medicamentos. $O$ estudo elaborado por Walker e colaboradores demonstra na prática essas ações. ${ }^{13}$ Esse estudo consistiu em criar uma prática padronizada de ATM em uma UTIN para neonatos submetidos a procedimentos cirúrgicos. Foram comparados os períodos anteriores e posteriores à implementação do protocolo de antibióticos em uma revisão retrospectiva. Como resultados, 158 lactentes foram submetidos a procedimentos cirúrgicos pré-protocolo e 127 foram submetidos a procedimentos pós-protocolo, as taxas de 
infecção do local cirúrgico foram semelhantes antes e após o protocolo, $14 \%$ e $9 \%$, respectivamente, $(P=0,21$.) e a incidência de infecções adquiridas no hospital foi $13,7 \%$ vs $8,7 \%(P=0,205)$. Assim, esse estudo demostrou que a utilização de antimicrobianos por muito tempo antes ou após as cirurgias não interfere no aumento de infecções. Portanto, a padronização possibilita uso racional de antimicrobianos evitando danos ao paciente e gerando economia para o hospital. ${ }^{13}$

Como demonstrado no estudo acima, a padronização do uso de ATM de forma ponderada e baseada em evidências pode ser realizada em neonatos, evitando assim utilização de ATM de maior espectro, aumento da resistência bacteriana e custos desnecessários.

Auditoria prospectiva de prescrição com intervenção

A auditoria prospectiva de prescrição de antimicrobianos é uma ação de intervenção em que consegue grande redução do uso inadequado de ATM. As auditorias têm por função verificar a indicação, posologia e duração do tratamento no momento da prescrição ou retrospectivamente. Deve ser realizada por profissionais capacitados, sendo que a equipe deverá ser composta por um infectologista, um farmacêutico clínico ou outro profissional com treinamento ou formação em doenças infecciosas ou no uso desses medicamentos. ${ }^{12}$

Denominadas também de revisão pósprescrição, as auditorias são realizadas através de visitas programadas, periódicas e conjuntas entre membros do time operacional e médicos assistenciais e residentes da unidade hospitalar. Durante as visitas acontece revisão e discussão de todos antimicrobianos em uso na unidade ou apenas dos estratégicos ou os de reserva. Para que realmente as auditorias sejam efetivas é necessário que todos os profissionais entendam claramente sua função ética e técnica, sendo fundamental a difusão do modelo interdisciplinar de decisão compartilhada. ${ }^{12}$

Um estudo realizado por Newland e colaboradores $^{14}$ em 2012 demonstra a importância e eficácia das auditorias. Nesse estudo cujos objetivos foram descrever uma metodologia prospectiva de auditoria com feedback em um hospital infantil independente e avaliar o impacto desse programa no atendimento ao paciente, demonstrou que após implementação das auditorias com a frequência de 3 vezes por semana houve reduções na utilização dos ATM. Como resultados, o estudo demonstrou que todo o uso de antibióticos diminuiu de 883 DoT por 1000 pacientes-dia antes da implementação do ASP para 787 DoT por 1000 pacientes-dia pósimplementação $\quad(P<0,001)$. Também, foi possível constatar que o uso total de antimicrobianos diminuiu de 567 LoT por 1000 pacientes-dia antes da intervenção para 523 LoT por 1000 pacientes-dia com o ASP instalado $(P<0,001)$. Como conclusão, o estudo menciona que a implementação das auditorias é viável, pois, permite a identificação de áreas para melhoria de qualidade direcionada, além de demonstrar redução no uso dos antimicrobianos sem afetar negativamente os pacientes. $^{14}$

A inserção de auditorias prospectivas não limita apenas ao consumo dos antimicrobianos, também é uma forma de avaliar medidas de resultados mais difíceis de quantificar, ou seja, aquelas que não são prontamente acessíveis à análise estatística tradicional. Como resultados futuros têm-se como principal objetivo a redução da resistência bacteriana e diminuição do consumo de ATM.

\section{Análise técnica das prescrições pela farmácia}

A farmácia clinica é crucial nos programas de gerenciamento de antimicrobianos. A atuação dos farmacêuticos clínicos engloba atividades nas quais os principais objetivos são melhorar a atenção ao paciente, consequentemente sua saúde e atuação em pró da efetivação dos indicadores propostos pelo programa. ${ }^{12}$

O farmacêutico clínico para atuar no gerenciamento dos antimicrobianos deve ser profissional dedicado, com formação adequada e conhecimento nessa área, para que assim, possa interagir com a equipe multiprofissional em todos os aspectos que englobem o tratamento do paciente. ${ }^{12}$

A atuação do farmacêutico clínico nos hospitais, especialmente em farmácias das unidades de terapia intensiva neonatais e pediátricas, ainda está em desenvolvimento no Brasil. Alguns estudos retratam a importância dos farmacêuticos cínicos nesses locais, como por exemplo um estudo realizado por Nunes e colaboradores $^{15}$ no ano de 2017 em uma UTI neonatal na cidade de Natal. Esse estudo teve como objetivo determinar os principais 
problemas relacionados a medicamentos em neonatos que utilizaram ATM. Como resultados, dos 152 neonatos incluídos na pesquisa foram identificados, em 51 dos pacientes, problemas relacionados a medicamentos (PRM), o que corresponde $33,6 \%$ dos pacientes. Os PRMs foram classificados como problemas potenciais, sendo que $84,8 \%$ destes estavam relacionados à efetividade do tratamento e $15,2 \%$ a reações adversas que foram relacionadas com a dose prescrita (subdose, sobredose e frequência de administração). ${ }^{15}$

É visível a necessidade de farmacêuticos clínicos em hospitais, devido principalmente sua capacidade em contribuir positivamente na redução de PRMs. A intervenção farmacêutica pode reduzir significativamente os erros de prescrição evitando assim os eventos adversos indesejáveis. ${ }^{15}$

A inserção do farmacêutico clínico na equipe multiprofissional é essencial para o acompanhamento dos pacientes, pois além de gerar economia financeira para a instituição, é uma barreira a mais para a proteção do paciente, além de evitar o uso exacerbado e desnecessário de ATM.

\section{Monitoramento do programa}

Após aplicação das ações é necessário que ocorra monitoramento para que assim seja constatada efetividade. Através do monitoramento é possível avaliar o impacto das intervenções para identificação de potenciais áreas de melhoria e promover o retorno das informações para todos os profissionais envolvidos.

Para realização do monitoramento são utilizados indicadores, sendo que são divididos em duas categorias, os indicadores ou medidas de processo que avaliam a qualidade na utilização de antimicrobianos e os indicadores ou medidas de desfecho/resultado que retratam a resistência microbiana, taxas de infecção por Clostridium difficile, resultados clínicos e redução de custos. ${ }^{12}$

Os indicadores de processo ou desempenho devem ser definidos de forma que possam apontar se as atividades propostas estão sendo bem executadas, já os indicadores de resultado e de impacto verificam se os objetivos propostos foram alcançados. Como exemplo de indicadores pode-se citar as medidas que avaliam o consumo de antimicrobianos. As medidas "Dias de Terapia"
(Days of therapy -DoT) e "Duração de Terapia" (Lenght of therapy - LoT) também são medidas que estão sendo adotadas, mostrando-se bastante úteis e relevantes. ${ }^{16}$

Atualmente não existe uma padronização da dose diária definida (DDD) em neonatologia, porém na literatura internacional alguns autores já propuseram esta padronização. Liem e colaboradores em 2010 realizaram um estudo com os dez ATM mais comumente utilizados em neonatologia com base em um peso neonatal presumido de 2 kg. Para validar o estudo os pesquisadores utilizaram oito fontes internacionais conceituadas na qual, seus dados fornecem as recomendações de dosagem de antibacterianos em crianças e recém-nascidos por suas indicações mais comuns. Com os resultados satisfatórios, os autores apontam que a DDDn é uma boa alternativa para mensurar o consumo de ATM na população neonatal, seja com o intuito para fins de pesquisa como também para que possa existir uma comparação entre diferentes unidades de saúde. ${ }^{17}$

\section{Divulgação dos resultados}

Outro ponto fundamental na implantação dos programas de gerenciamento de antimicrobianos é a divulgação dos resultados. Pois, com análise de dados do monitoramento pode ser demonstrado o impacto do programa sobre os resultados clínicos, a resistência bacteriana aos antimicrobianos e os custos de cuidados de saúde do hospital, além de instigar outras melhorias nas ações das equipes de saúde.

\section{Considerações finais}

Os antimicrobianos são medicamentos essenciais e estão entre os mais prescritos nas UTIs neonatais, sendo que a implantação de programas de gerenciamento de antimicrobianos é crucial para o uso racional de ATM além de diminuir a resistência bacteriana.

\section{Referências}

1. Tzialla C, Borghesi A, Perotti GF, Garofoli F, Manzoni P, Stronati M. Use and misuse of antibiotics in the neonatal intensive care unit. The Journal of Maternal-Fetal \& Neonatal Medicine. 2012; 25(4): 2729.Disponível em: https://doi.org/10.3109/14767058.2012.71498 $\underline{7}$. 
2. Prusakov P, Goff DA, Wozniak PS, Cassim A, Scipion CE, Urzúa S.Global NEO-ASP study group.prusakov. A global point prevalence survey of antimicrobial use in neonatal intensive care units: The no-moreantibiotics and resistance (NO-MAS-R) study. EClinicalMedicine. 2021;(32):100727. Disponível em: https://doi.org/10.1016/j.eclinm.2021.100727. 3. Silva ERM. Análise do perfil das prescrições de antimicrobianos na clínica médica de um hospital público do Pará. Revista Brasileira de Farmácia Hospitalar e Serviços de Saúde. 2012;3(2). Disponível em: https://rbfhss.org.br/sbrafh/article/view/125.

4. Simon A, Zemlin M, Geipel M, Gärtner B, Armann J, Meyer S. Hygienestandards auf einer neonatologischen Intensivstation. Der Gynäkologe. 2021; 1-6. Disponível em: https://link.springer.com/article/10.1007/s001 29-021-04804-6.

5. Ting JY, Roberts A, Tilley P, Robinson JL, Dunn MS, Paquette V, Shah PS. Development of a national neonatal intensive care unit-specific antimicrobial stewardship programme in Canada: protocol for a cohort study. BMJ open. 2020;10(12):e043403. Disponível em: https://bmjopen.bmj.com/content/10/12/e043 403.abstract.

6. Sánchez PJ, Moallem $M$, Cantey JB, Milton A, Michelow I. Empiric therapy with vancomycin in theneonatal intensive care unit: let's "get smart" globally! Jornal de pediatria. 2016; 92(5):432-435. Disponível em: https://www.scielo.br/pdf/jped/v92n5/pt_002 1-7557-jped-92-05-0432.pdf.

7. Cantey JB, Wozniak PS, Pruszynski JE, Sánchez P. Reducing unnecessary antibiotic use in the neonatal intensive care unit (SCOUT): a prospective interrupted time-series study. The Lancet Infectious Diseases. 2016; 16(10):11781184.Disponível em: https://doi.org/10.1016/S1473-3099(16)30205$\underline{5}$.

8. Locatelli DL. Perfil de antimicrobianos utilizados em uma unidade de tratamento intensivo neonatal de um hospital materno infantil. Porto Alegre: Faculdade de farmácia, Universidade Federal do Rio Grande do Sul; 2017.

9. Gkentzi D, Dimitriou G. Antimicrobial stewardship in the neonatal intensive care unit: an update. Current pediatric reviews. 2019;15(1): 47-52.Disponível em: https://doi.org/10.2174/15733963156661 $\underline{90118101953 .}$.
10. Barlam TF, Cosgrove SE, Abbo LM, MacDougall C, Schuetz AN, Septimus EJ, Trivedi K. Implementing an antibiotic stewardship program: guidelines by the Infectious Diseases Society of America and the Society for Healthcare Epidemiology of America. Clinical infectious diseases. 2016;62(10):51e77.Disponível em: https://doi.org/10.1093/cid/ciw118.

11. World Health Organization. Antimicrobial stewardship programmes in health-care facilities in low-and middle-income countries: a WHO practical toolkit. 2019. Disponível em: https://apps.who.int/iris/bitstream/handle/106 65/329404/9789240003095-rus.pdf.

12. Brasil. Diretriz Nacional para Elaboração de Programa de Gerenciamento do Uso de Antimicrobianos em Serviços de Saúde. Brasília DF: Ministério da Saúde; 2017.

13. Walker S, Datta A, Massoumi RL, Gross $E R$, Uhing $M$, Arca MJ. Antibiotic stewardship in the newborn surgical patient: A quality improvement project in the neonatal intensive care unit. Surgery. 2017; 162(6):1295-1303. Disponível em: https://doi.org/10.1016/j.surg.2017.07.021. $14 . \quad$ Newland JG, Stach LM, De Lurgio SA, Hedican E, Yu D, Herigon JC, Zaoutis TE. Impact of a prospective-audit-with-feedback antimicrobial stewardship program at a children's hospital. Journal of the Pediatric Infectious Diseases Society. 2012;1(3):179-186. Disponível em: https://doi.org/10.1093/jpids/pis054.

15. Nunes BM, Xavier TC, Martins RR. Problemas relacionados a medicamentos antimicrobianos em unidade de terapia intensiva neonatal. Revista Brasileira de Terapia Intensiva. 2017;29(3):331-336. Disponível em: https://doi.org/10.5935/0103-507x.20170040. 16. World Health Organization. DDD indicators [internet]. Disponível em: https://www.whocc.no/atc_ddd_index/.

17. Liem TB, Heerdink ER, Egberts AC, Rademaker CM. Quantifying antibiotic use in paediatrics: a proposal for neonatal DDDs. Eur J Clin Microbiol Infect Dis. 2010;29(10):1301-3. Disponível:https://link.springer.com/article/10. 1007/s10096-010-0990-3.

18. RODRIGUES, A. M. V. N.; MANCINI, M. C.; VAZ, D. V.; SILVA, L. C. Uso de órteses para abdução do polegar no desempenho funcional de criança portadora de paralisia cerebral: Estudo de caso único. Rev. Bras. Saúde Matern. Infant., Recife, v. 7, n. 4, p. 423-436, 2007. 
Endereço para Correspondência

Cinara Rejane Vieira Oliveira

Universidade do Estado da Bahia

Rua Silveira Martins, 2555 - Cabula

Salvador/BA, Brasil

CEP: 41192-010

E-mail: cinararejane26@gmail.com

Recebido em 03/03/2021

Aprovado em 22/05/2021

Publicado em 27/09/2021 